\title{
Path Selection and Adaptive Selection of Smart Antenna Transmission Schemes in Multi-hop Wireless Networks
}

\author{
Muhammad Irfan Rafique and Thomas Bauschert \\ Chair for Communication Networks, \\ Chemnitz University of Technology, \\ Chemnitz, Germany \\ \{irfan.rafique, thomas.bauschert\} detit.tu-chemnitz.de
}

\begin{abstract}
The performance of multi-hop wireless networks can be substantially improved by using smart antennas. However, the utilization of smart antennas is quite challenging as the benefit of the different transmission modes strongly depends on specific channel conditions. In this paper we present a novel path selection protocol PSPSA (path selection protocol for smart antennas) and a modified MAC protocol that exploits the benefits of smart antennas. PSPSA selects an optimum path considering the specific ranges and transmission rates implied by the different transmission schemes (spatial multiplexing and beamforming). The MAC protocol is a modification of the traditional DCF scheme to cope with the different PHY layer transmission schemes. Our solution enables mesh nodes to dynamically select their transmission scheme on a per packet basis according to the current channel conditions. Simulation results show that the overall packet success rate (throughput) of multi-hop wireless networks are substantially improved.
\end{abstract}

Keywords: Cross Layer Approach, Wireless Mesh Networks, Smart Antennas, Path Selection Protocol, MAC Layer.

\section{$1 \quad$ Introduction}

In the last decade multi-hop wireless networks have captured a great importance due to their diverse applications. However the multi-hop structure and the uncertainty of the wireless channel are critical performance limiting factors. The amendments at PHY layer proposed in IEEE 802.11n [1] could increase the efficiency of multi-hop networks but the incorporation of smart antenna features is quite challenging due to the mutual influence of the transmission scheme and the MAC mechanism. Furthermore the choice of the transmission scheme strongly depends on the current network topology and channel conditions, see [4]. For example, beamforming enables directional transmission with extended range thus being suitable for sparse network topologies whereas spatial multiplexing enables high bit rate omni-directional transmission with lower range thus exploiting advantages in dense networks. Thus, a transmitting node might also influence a different number of neighboring nodes depending on whether beamforming or spatial multiplexing is used. Hence it is advisable that path selection protocol, MAC mechanisms and PHY layer transmission techniques are operating in a well aligned manner. 
In this paper we consider stationary multi-hop networks like wireless mesh networks (WMNs). We present a cross layer approach of a novel path selection protocol and a modified channel access mechanism that considers the adaptively (per packet) selected transmission scheme at PHY layer. Our novel path selection protocol PSPSA works similar to the reactive base mechanism of HWMP [2] but exploits the advantages of smart antennas using multiplexing and beamforming transmission according to the channel conditions. Our MAC protocol is based on the basic channel access mechanism of the traditional (DCF) MAC and includes some modifications to cope with different $\mathrm{PHY}$ transmission schemes.

We assume that VBLAST [3] is applied for spatial multiplexing and the technique relying on angle of arrival (AOA) is used for beamforming. The antenna gain for bidirectional beamforming is determined according to the formula (1) where $M$ and $N$ denote the number of antenna elements at the transmitter and receiver side [4]:

$$
G_{\text {array }}=(\sqrt{M}+\sqrt{N})^{2}
$$

The paper is organized as follows: Section II describes the related work. Section III addresses the modifications of the MAC protocol whereas in Section IV the PSPSA path selection protocol is explained. The results of our simulations are discussed in Section V. Section VI concludes the paper.

\section{$2 \quad$ Related Work}

A number of directional routing protocols for ad hoc networks have been proposed in the last years but these consider only beamforming realized by specific directional antennas, see [5]-[12]. The MIR [13] routing protocol is the first one which incorporates MIMO features like multiplexing and diversity but neither considers beamforming nor adaptive selection of the transmission scheme wrt. channel conditions. In [14] a routing protocol is presented that combines the advantages of multiplexing gain and interference cancellation. Lin et al. [15] proposes an algorithm that coordinates the scheduling, routing and power control mechanisms. Cai et al. [16] enhances an AODV-based routing protocol to consider spatial multiplexing at the PHY layer and focuses on the minimization of the route establishment time. Hu and Zhang [17] propose an extension of the IEEE 802.11 MAC protocol that is aware of spatial diversity and analyzes its impact on the routing. In Xu et al. [18] propose a tree based interference aware scheduling and routing algorithm for wireless backhaul networks with smart antennas.

In our previous work, we introduced MHWMP which is a modification of the path selection protocol HWMP [2] to apply smart antennas in mesh networks. To our knowledge it was the first attempt to incorporate full features of smart antennas in mesh networks. Simulation results show that MHWMP outperforms HWMP in terms of transmission rates and robustness. MHWMP applies spatial multiplexing to achieve high transmission rates and falls back to beamforming during congestion. However it increases the routing protocol overhead due to two separate paths discoveries (for beamforming and multiplexing transmission, respectively). Additionally, in scenarios where some links on the path between source and destination do not allow for spatial multiplexing transmission, MHWMP applies beamforming transmission on all links on the path, irrespective to the channel conditions and the mesh network topology. This is illustrated in Fig. 1 where with MHWMP on all links of the path S-X-Y-D 
beamforming is applied regardless the fact that link $\mathrm{X}-\mathrm{Y}$ would allow for spatial multiplexing. In the following, as foundation for understanding PSPSA, MHWMP is described briefly. For more details interested readers are referred to [19].

In MHWMP each node has two path tables - an Omnidirectional Path Table (OPT) and a Directional Path Table (DPT). The former is used for communication with spatial multiplexing while the latter is considered for communication with beamforming. When a node does not have a valid path entry for a specific destination in both path tables, it broadcasts two path requests with standard omnidirectional antenna (O-PREQ) and with spatial diversity (S-PREQ, [20]) to gain the missing OPT and DPT path entries, respectively. On receiving a PREQ, the destination node sends a path reply with omnidirectional antenna transmission (O-PREP) and with beamforming transmission (D-PREP), respectivelly. Intermediate nodes then update their corresponding path tables. If an valid entry exists in one of the path tables (OPT or DPT), the source node begins sending data frames using the respective transmission scheme and starts a path discovery to gain the missing path entry in the other table. If a path is availabe in both path tables then the source selects the best path according to the applied metric and uses the corresponding transmission scheme for the data transmission. When a link outage during data transmission with spatial multiplexing occurs, the node that detects the outage sends a path error frame (PERR) towards the source. Meanwhile it continues the transmission of queued packets with beamforming on the path available in DPT. By receiving a PERR frame, the source generates an OPREQ to find a new path which is suitable for multiplexing transmission. If an valid entry exists in the DPT, the source continues transmission with beamforming until a new path suitable for multiplexing transmission is found.

The new path selection protocol PSPSA which we describe in this paper decreases the overhead compared to MHWMP by using a single PREQ for path discovery and allowing hybrid transmission paths i.e. paths with different PHY layer transmission schemes applied on the links. Furthermore, to cope with the adaptivity of the PHY layer transmission, some modifications of the MAC scheme are proposed.

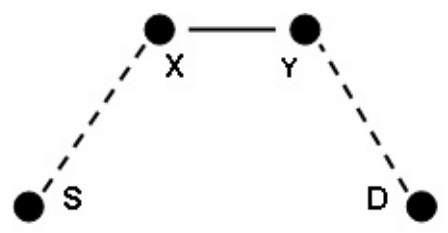

Fig. 1.

\section{MAC Protocol Modification}

In this chapter we describe the amendments regarding the RTS/CTS mechanism in our modified MAC protocol.

As the transmission range of beamforming is different compared to omni-directional antenna transmission, the standard RTS/CTS mechanism (which originally assumes omni-directional antenna mode) has to be modified. In [19] the diversity gain achieved by STBC [20] was utilized to extend the communication range when sending RTS/CTS frames prior to data transmission with beamforming. However, in realistic scenarios the diversity gain heavily depends upon the nature of the environment 
(uncorrelated and correlated radio propagation conditions), see e.g. [21]-[24]. In our approach, the RTS/CTS frames are transmitted with higher and normal transmit power prior to data transmission with beamforming and multiplexing, respectively. Furthermore a tag is attached to the RTS/CTS frames in order to inform the receivers about the transmission mode of data and ack frames (one bit is sufficient for this purpose). The transmission power of RTS/CTS frames prior to data transmission with beamforming is set so as to exactly match the range of beamforming transmission under ideal conditions (line of sight conditions). The transmission range of standard omni-directional antenna transmission is assumed to be equal to that of multiplexing transmission. DNAV [25] is applied as well to allow parallel communication with beamforming. After successful reception of RTS/CTS frames for beamforming both transmitter and receiver steer their beams to each other for data and ack frames and put nulls to other directions

\section{$4 \quad$ Path Selection Protocol PSPSA}

\subsection{Neighbor Discovery (ND)}

As the transmission ranges for beamforming and multiplexing are different, the neighbors a node discovers might be different for these two schemes. Therefore, like in MHWMP, in PSPSA each node also has two neighboring tables - the Omni-directional Neighbor Table (ONT) and the Directional Neighbor Table (DNT). A neighbor node entry in ONT and DNT means that the link to this neighbor node might be valid for multiplexing and beamforming transmission, respectively. In DNT the neighbors are stored together with their respective directions. To find the omni-directional and the directional neighbors, beacons are broadcasted with normal and with higher transmission power, respectively. A tag is attached to differentiate the two types of beacons. We propose to transmit the beacons alternately in 0.5 second intervals.

\subsection{Path Selection Metric}

The optimum path from source to destination is determined based on the airtime link metric (AM). The airtime link metric is defined as follows:

$$
C=\left[O_{c a}+O_{P}+\frac{B_{t}}{r}\right] \frac{1}{1-e_{f r}}
$$

where $O_{c a}$ and $O_{p}$ are the channel access and MAC protocol overheads, $B_{t}$ is the number of bits of a test frame and $e_{f r}$ denotes the frame error probability. $\mathrm{r}$ is the PHY layer transmission rate determined by the rate adaptation algorithm RBAR [26] that takes into account the channel conditions. If spatial multiplexing is applied on a link, $r$ has to be replaced by $r_{\text {mux }} . r_{\text {mux }}$ is calculated as follows:

$$
r_{\text {mux }}=\min (M, N) r
$$

where $M$ and $N$ denote the number of antennas in the antenna array of the transmitter and receiver, respectively. 


\subsection{Path Discovery}

When a source $\mathrm{S}$ wants to communicate with destination $\mathrm{D}$, it looks in its path table. In case $\mathrm{S}$ does not find any entry for $\mathrm{D}$, it broadcasts path request (PREQ) frames with higher transmission power. PREQ frames have the same structure as in HWMP. If an intermediate node receives a PREQ it looks into its neighbor tables. If the node that sent the PREQ exists in both ONT and DNT, the link is valid for both transmission schemes (multiplexing and beamforming). The node calculates the airtime link metrics considering both schemes and stores the next hop node towards the source (and the respective transmission scheme) that lies on the path with the lowest airtime metric in its path table. In case that in the path table already a valid entry for the destination D exists, the node replies with the corresponding transmission scheme and does not forward the PREQ. Otherwise it updates its path table and forwards the PREQ (with higher transmission power) only if its sequence number is higher or, in case of equal sequence numbers, if the airtime metric value contained in the PREQ is better than that of the corresponding path entry in the path table. When the destination node receives a new PREQ it sends a Path Reply (PREP) on the return path towards the source applying a transmission mode accordingly. The processing of PREP frames in an intermediate node works similar to original HWMP except that PREP frames are now transmitted with the specific transmission scheme on the link towards the PREP receiver.

\subsection{Path Maintenance}

We assume a stationary multi-hop network (i.e. a network with non-moving nodes) where link outages might only occur due to bad radio channel conditions, i.e. poor SNR or when nodes are powered off. Path discoveries after the failure of links (due to insufficient SNR) that apply multiplexing can be avoided by switching to beamforming transmission, thus reducing the protocol overhead. In our approach a data frame is sent with beamforming transmission after seven unsuccessful retransmissions with multiplexing. In case a data frame is not received successfully even after seven retransmissions (applying beamforming) the data frame is dropped and a PERR frame is generated. In case the data frame is successfully received (applying beamforming), the node perceives, that the link failure happened due to insufficient channel conditions for multiplexing and all further transmissions can take place with beamforming. Switching from multiplexing to beamforming on a link increases the airtime metric of end-to-end paths that include this link (as beamforming allows less transmission rate $r$ ). As a source chooses the path with the lowest airtime metric among available paths, this switching could result in a new optimum path. Therefore, a node which changes the transmission scheme on any of its links should inform the affected sources. For that, we propose to introduce a new control frame called Switching Transmission Scheme Info (STSI) that is to be sent with higher transmission power towards the sources. Intermediate nodes that receive a STSI frame, increase the switch value of the corresponding path entry and forward the STSI towards the source (with higher transmission power). When a source receives a STSI, it updates its corresponding switch value and, if this value exceeds a threshold, it starts a path discovery to find a new path between source to destination. Otherwise it 
continues its transmission on the current path applying the same transmission scheme until the lifetime of the path ends. The threshold could be set according to traffic requirements. The switch value is set to zero whenever a path is established after a path discovery.

In case a link outage occurs while sending data frames with beamforming, the node that recognizes the link outage sends (with higher transmission power) a PERR frame towards the source. Intermediate nodes that receive the PERR frame delete the corresponding entries in their path tables and forward the PERR frame towards the source. The high transmission power of PERR frames helps to avoid a wrong airtime metric calculation at node $y$ for the next PREQ send by node $x$ in case that before the failure of link $x-y$ multiplexing transmission has been used.

\section{$5 \quad$ Performance Evaluation}

The performance analysis of a wireless mesh network on which our new concepts are applied is carried out using the network simulator ns-3. We assume constant bit rate (CBR) traffic flows between source and destination nodes and a constant data packet size of 512 bytes. Each node is assumed to be equipped with a uniform circular antenna array comprising of four antenna elements. For simulation of beamforming,

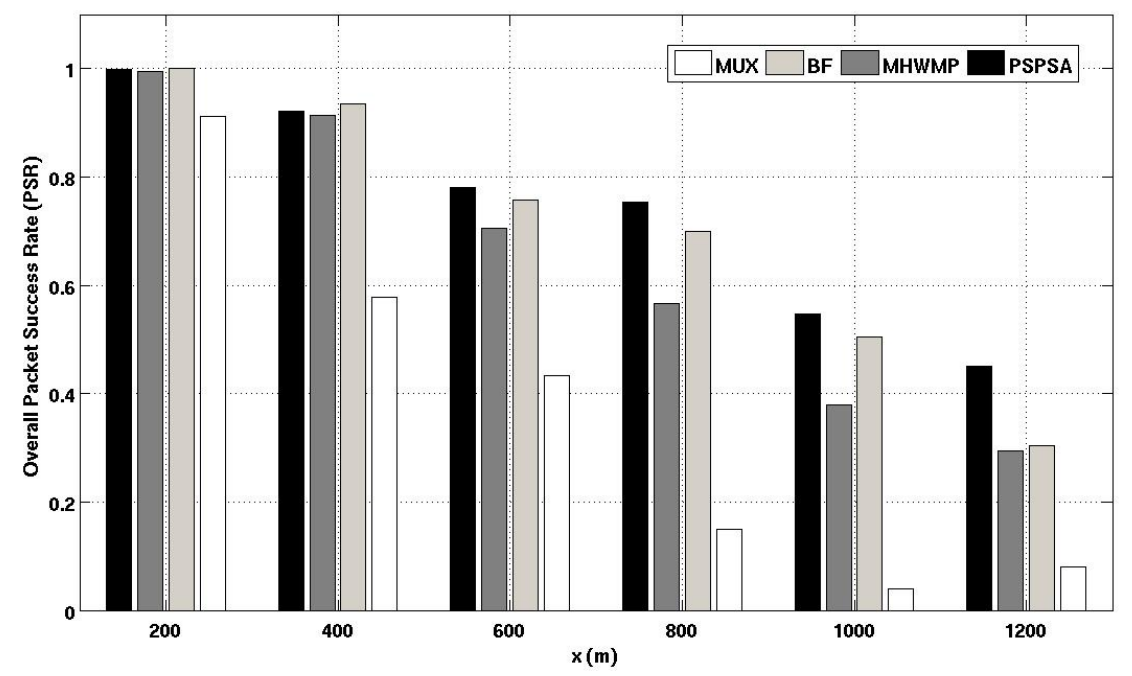

Fig. 2. Overall Packet Success Rate (stable channel conditions)

the keyhole model is applied where the gain of the side lobes is set equal to zero. The maximum net transmission rate for a single antenna element is $54 \mathrm{Mbps}$ (according to IEEE 802.11a). The lifetime of the path table entries is assumed to be 3 seconds. Results from previous simulations show, that with these parameter settings, two nodes applying spatial multiplexing are able to communicate effectively with each other if their distance does not exceed $175 \mathrm{~m}$. 
The performance of our approach PSPSA is compared to that of MHWMP and HWMP applying beamforming (BF) and multiplexing (MUX). Each simulation is carried out multiple times and the shown results represent average values wrt. these runs. Performance indicators are the overall packet success rate (PSR) and the overall protocol overhead. The PSR is defined as the ratio of received data frames at the destination to transmitted frames at the source and the overall protocol overhead is defined to be the number of control frames received by all nodes in the network.

In our first simulation scenario, we consider stable channel conditions where no degradations of the links (SNR) occur. Five active node pairs are selected randomly among 20 nodes placed at random locations in a square field of varying side length $x$. Each source generates (UDP) traffic at constant rate of 1Mbps. Fig. 2 illustrates the overall PSR of the network. It can be observed that the performance of all path selection schemes decrease as $x$ is increased. This can be explained as follows: when $\mathrm{x}$ is increased, the connectivity of nodes decreases leading to a higher number of multi-hop communications and an increased level of mutual interference in the mesh network. Additionally, longer links exhibit a lower SNR compared to short links and the rate adaptation algorithm chooses a lower (PHY layer) transmission rate, which in turn make these links prone to congestion. For increasing $x$ PSPSA performs best while HWMP with spatial multiplexing transmission (MUX) always shows worst behavior. The bad performance of MUX especially for larger $x$ is because more and

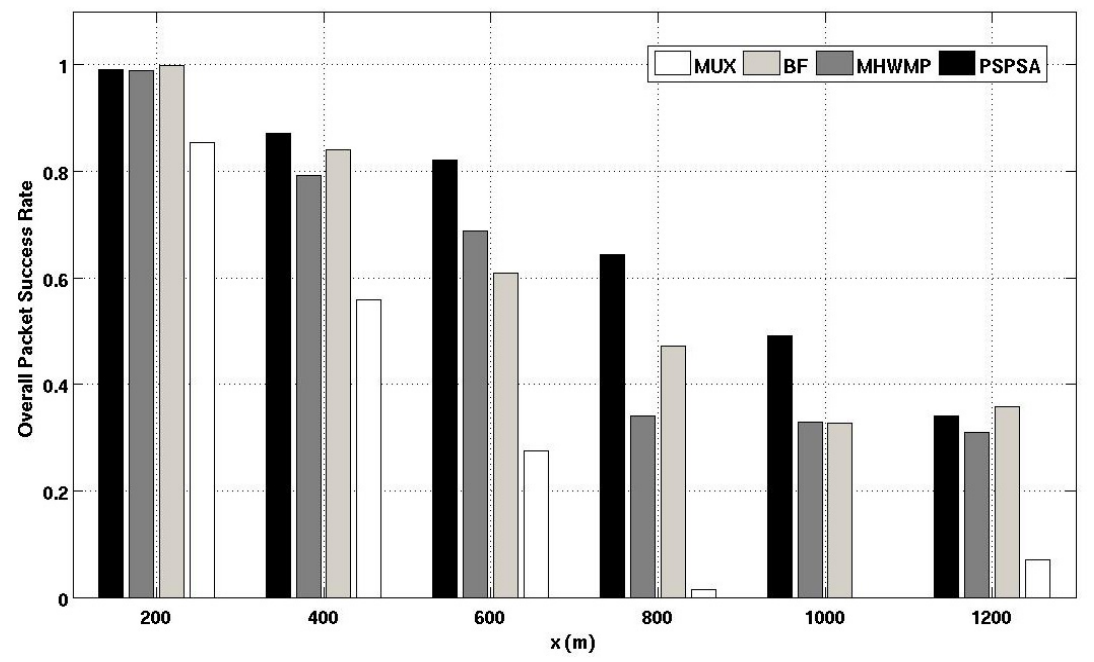

Fig. 3. Overall Packet Success Rate (with random link degradations)

more links cannot be maintained anymore with spatial multiplexing and the connectivity declines. The other schemes are less sensitive as they enable nodes to establish links with beamforming transmission. MHWMP shows poor performance compared to PSPSA and BF when x exceeds 400m. This can be explained as follows: as MHWMP sends two PREQs for path discovery the probability of MAC collisions is higher than for PSPSA and HWMP with beamforming (BF) leading to a slightly less data throughput (and PSR, respectively) compared to these schemes. 
In a second simulation scenario, we also consider link degradations. Once again five active node pairs are selected randomly among 20 nodes placed at random locations in a square field of side length $x$. For five randomly selected nodes the quality of their links (in incoming and outgoing direction) is deteriorated by reducing the SNR by $16 \mathrm{~dB}$ at random time instances. The deterioration time period is set to 0.5s. Fig. 3 illustrates the PSR performance. It can be observed that overall PSR now is worse compared to Fig. 2. The major reason of this performance degradation are link failures. PSPSA again shows best performance compared to the other schemes as it avoids path discoveries (after links operated with multiplexing fail due to insufficient SNR) by switching to beamforming for further transmissions. In contrast, the other schemes generate PERR frames that in turn lead to new route discoveries. Thus more control frames are generated increasing the probability of MAC collisions and resulting in less data throughput (and lower PSR, respecively). Hence the performance gap of MHWPM, MUX and BF compared to PSPSA is larger for random link degradation (Fig. 3) than for stable channel conditions (Fig. 2).

Fig. 4 illustrates the overall protocol overhead of the different path selection schemes depending on the field side length $\mathrm{x}$ for both scenarios. Both PSPSA and MHWMP normally induce higher overhead than standard HWMP (MUX and BF). This is due to the fact that in PSPSA and MHWMP, two types of control frames are

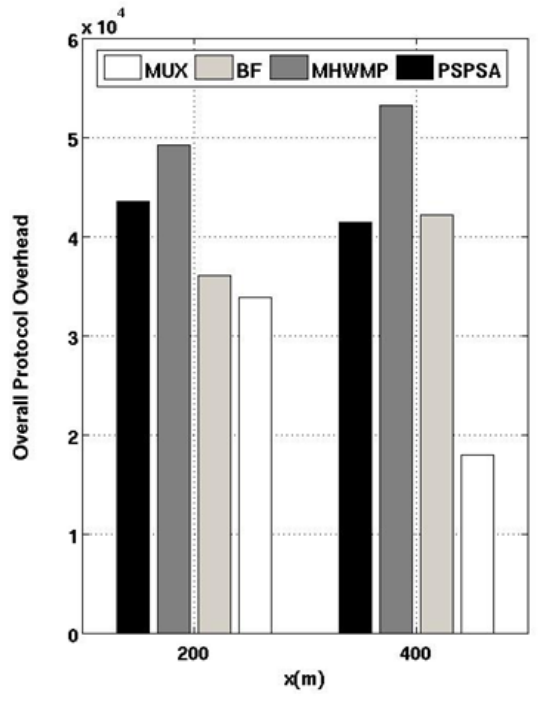

a) Stable Channel Conditions

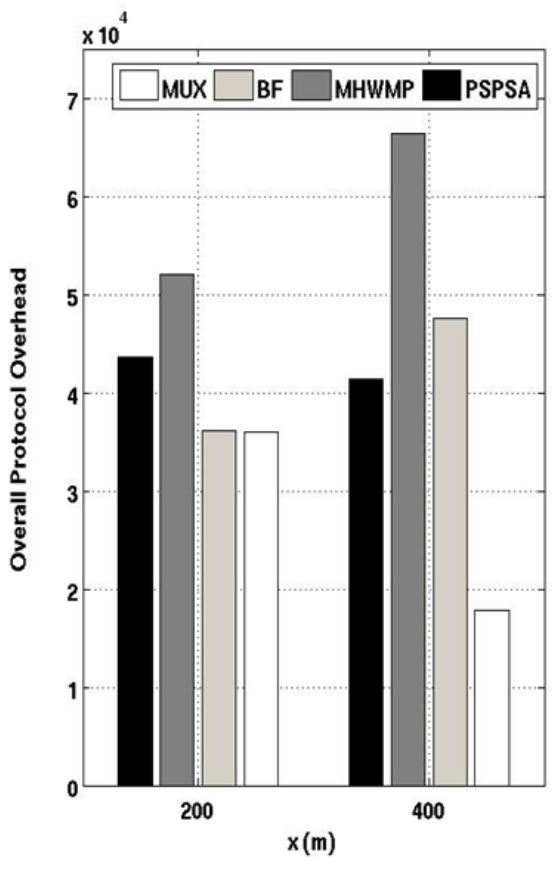

(b) Random Link Degradations

Fig. 4. Overall Protocol Overhead 
exchanged between nodes to establish links to neighbors that can be reached by multiplexing and beamforming transmission. MUX generates the lowest overhead compared to the other schemes. However this comes at the cost of low connectivity and low throughput/PSR (see Fig. 2 and Fig. 3). MHWMP always generates the highest overhead due to its two separate path discoveries.

Fig. 4a shows the protocol overhead in case of stable channel conditions. As $\mathrm{x}$ is increased from $200 \mathrm{~m}$ to $400 \mathrm{~m}$, more multi-hop communications take place increasing the probability of MAC layer collisions and data and control frame retransmissions. For MHWMP and BF the number of control frames increase due to new route discoveries. PSPSA partially avoids these control frames by simply switching to beamforming transmission on links where originally multiplexing was used. For MUX the number of control frames drops as several neighbors cannot be reached anymore at $\mathrm{x}=400 \mathrm{~m}$.

Fig. $4 \mathrm{~b}$ shows the overall protocol overhead in case of random link degradations. Compared to the situation with stable channel conditions (Fig. 4a) the overhead remains quite unchanged in case of PSPSA whereas it increases for MHWMP, BF and MUX. PSPSA successfully switches to beamforming on degraded links and thus avoids starting new path discoveries. On the other hand MHWMP and BF start new path discoveries which increases the overhead. MUX shows less overhead due to the reduced connectivity.

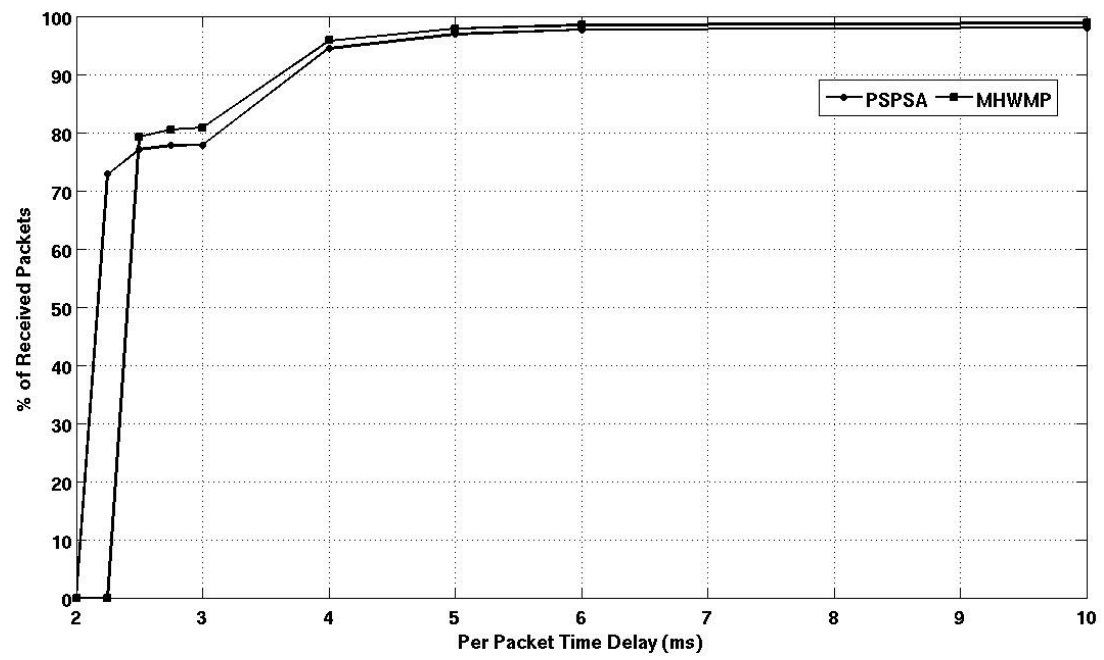

Fig. 5. End-to-End Packet Delay Time Distribution

Finally, to assess the performance advantages of PSPSA in cases where different transmission schemes might be applied on the links of one path, the simple network scenario depicted in Fig. 1 is simulated for PSPSA and MHWMP. Here the source node $\mathrm{S}$ generates traffic at a constant rate of $500 \mathrm{Kbps}$. The simulation results show that for both path selection schemes the destination node $\mathrm{D}$ successfully receives all transmitted data packets, i.e. the PSR turns out to be 1. Fig. 5 shows the distribution function of the per packet delay time. For PSPSA, more than 70\% of the packets 
suffer a delay less than $2.25 \mathrm{~ms}$ while the packet delay for MHWMP is slightly higher. A detailed analysis shows that on link X-Y, PSPSA applies spatial multiplexing with $72(=18.4)$ Mbps transmission rate and MHWMP applies beamforming with $36 \mathrm{Mbps}$ transmission rate, respectively. Both algorithms (due to DNAV) allow for parallel communication on the links S-X and Y-D using beamforming and $6 \mathrm{Mbps}$ transmission rate.

Since for PSPSA the transmission rate on link X-Y is twice that of MHWMP, only half of the time is required for the transmission of data and ack frames. Therefore in PSPSA, the end to end delay of most packets is slightly less compared to MHWMP. One can expect that the performance improvements of PSPSA compared to MHWMP will be more significant for large mesh networks where advantageous (in terms of the air-time cost metric) multi-hop paths with different transmission schemes on the links (which can be used only by PSPSA) exist with higher probability. Note that the packet delay performance of BF will be quite similar to MHWMP (as beamforming is applied on all links along the S-D path) while MUX will not work at all as the links S$\mathrm{X}$ and $\mathrm{Y}-\mathrm{D}$ cannot be operated with multiplexing due to their lengths.

\section{Conclusion}

In this paper we describe and analyze a framework consisting of a path selection protocol (PSPSA) and a modified MAC protocol that leverages the advantages of smart antenna transmission schemes in multi-hop wireless networks. PSPSA considers both transmission schemes (multiplexing and beamforming) in the path selection process and may generate hybrid paths where different transmission schemes are applied on the links of the path. In case of link quality degradations (poor SNR) it avoids link outages by simply switching from multiplexing to beamforming transmission. The traditional MAC mechanism (DCF) has been modified to make it adaptive to the PHY layer transmission schemes. Simulation results show that PSPSA decreases the overhead compared to our previously proposed path selection scheme MHWMP and increases the overall packet success rate (PSR). In our future work we plan to evaluate the performance of PSPSA with further enhanced MAC mechanisms and for mesh networks of large size.

\section{References}

1. IEEE P802.11n, Part 11: Wireless Medium Access Control (MAC) and Physical layer (PHY) specifications, Amendment 5: Enhancement for higher throughput (2009)

2. IEEE 802.11 Part 11: Wireless Medium Access Control (MAC) and Physical layer (PHY) specifications, Amendment 10: Mesh Networking (2011)

3. Wolniansky, P.W., Foschini, G.J., Golden, G.D., Valenzuela, R.A.: V-blast: an architecture for realizing very high data rates over the rich-scattering wireless channel. Signals, Systems, and Electronics, 295-300 (1998)

4. Sundersasan, K.: Network Protocols for Ad-Hoc Networks with Smart Antennas, PhD thesis, Georgia Institute of Technology, USA (2006)

5. Choudhury, R.R., Vaidya, N.H.: Impact of Directional Antennas on Ad Hoc Routing. In: Conti, M., Giordano, S., Gregori, E., Olariu, S. (eds.) PWC 2003. LNCS, vol. 2775, pp. 590-600. Springer, Heidelberg (2003) 
6. Hu, B., Gharavi, H.: Directional routing protocol for ad hoc networks. IET Communications 2(5), 650-657 (2008)

7. Roy, S., Saha, D., Bandyopadhyay, S., Ueda, T., Tanaka, S.: A network-aware MAC and routing protocol for effective load balancing in ad hoc wireless networks with directional antenna. In: 4th ACM International Symposium on Mobile Ad Hoc Networking \& Computing, Maryland, USA (2003)

8. Gossain, H., Joshi, T., De M. Cordeiro, C., Agrawal, D.P.: DRP: An efficient directional routing protocol for mobile ad hoc networks. IEEE Trans. Parallel and Distributed Systems 17(12), 1438-1451 (2006)

9. Kang, I., Poovendran, R.: Design issues on broadcast routing algorithms using realistic cost-effective smart antenna models. In: IEEE VTC 2004, 2121-2125 (2004)

10. Nasipuri, A., Mandava, J., Manchala, H., Hirmoto, R.E.: On-demand routing using directional antennas in mobile ad hoc networks. In: IEEE ICCCN 2000 (2000)

11. Hu, B., Gharavi, H.: Directional routing protocol for ad hoc networks. IET Communications 2(5), 650-657 (2008)

12. Saha, A.K., Johnson, D.B.: Routing improvement using directional antennas in mobile ad hoc networks. In: IEEE GLOBALCOM 2004 (2004)

13. Sundaresan, K., Sivakumar, R.: Routing in ad-hoc networks with MIMO links: Optimization considerations and protocols. Computer Networks 52(14), 2623-2644 (2008)

14. Mundarath, J.C., Ramanathan, P., Van Veen, B.D.: A quality of service aware cross layer approach for wireless ad hoc networks with smart antennas. Ad Hoc Networks 7(5), 891903 (2009)

15. Lin, Y., Javidi, T., Cruz, R.L., Milstein, L.B.: Distributed link scheduling, power control and routing for multi-hop wireless MIMO networks. In: IEEE Asilomar Conference on Signals, Systems, and Computers, pp. 122-126 (2006)

16. Cai, X., Li, J., Chen, D., Zhang, Y.: Cross-layer design of aodv-based routing in ad hoc networks with MIMO links. In: Personal, Indoor and Mobile Radio Communications, pp. 2586-2590 (2009)

17. Hu, M., Zhang, J.: MIMO ad hoc networks: medium access control, saturation throughput, and optimal hop distance. Journal of Communication and Networks, 317-330 (2004)

18. Xu, Y., Wan, S., Tang, J., Wolff, R.S.: Interference aware routing and scheduling in wireless backhaul networks with smart antennas. In: IEEE SECON 2009, pp. 1-9 (2009)

19. Rafique, M.I., Porsch, M., Bauschert, T.: Modified HWMP for wireless mesh networks with smart antennas. In: IEEE GLOBECOM 2011 (2011)

20. Alamouti, S.M.: A simple transmit diversity technique for wireless communications. IEEE JSAC 16(8), 1451-1458 (1998)

21. Shiu, D.-S., Foschini, G.J., Gans, M.J., Kahn, J.M.: Fading correlation and its effect on the capacity of multielement antenna systems. IEEE Transactions on Communications 48(3), 502-513 (2000)

22. Bolcskei, H., Gore, D.A., Gesbert, D., Paulraj, A.J.: Outdoor MIMO wireless channels: models and performance prediction. IEEE Transactions on Communications 50(12), 19261934 (2002)

23. Jayaweera, S.K., Poor, H.V.: On the capacity of multiple-antenna systems in rician fading. IEEE Transactions on Wireless Communications 4(3), 1102-1111 (2005)

24. Kang, M., Alouini, M.S.: Capacity of MIMO rician channels. IEEE Transactions on Wireless Communications 5(1), 112-122 (2006)

25. Takai, M., Martin, J., Bagrodia, R., Ren, A.: Directional virtual carrier sensing for directional antennas in mobile ad hoc networks. In: 3rd ACM International Symposium on Mobile ad Hoc Networking \& Computing, MobiHoc 2002, New York, USA, pp. 183-193 (2002)

26. Holland, G., Vaidya, N., Bahl, P.: A rate-adaptive MAC protocol for multi-hop wireless networks. In: MobiCom 2001, New York, USA, pp. 236-251 (2001) 